\title{
Ocupação de conchas de gastrópodes por ermitões (Decapoda, Anomura) no litoral de Rio Grande, Rio Grande do Sul, Brasil
}

\author{
Luciane Ayres-Peres ${ }^{1}$, Carolina C. Sokolowicz ${ }^{1}$, Carla B. Kotzian ${ }^{2}$, Paulo J. Rieger ${ }^{3} \&$ \\ Sandro Santos ${ }^{1}$
}

1. Laboratório de Carcinologia, Departamento de Biologia, Universidade Federal de Santa Maria, 97.105-900 Santa Maria, RS. (lucianeayres@hotmail.com; carolinasokolowicz@hotmail.com; ssantos_sm@yahoo.com.br)

2. Laboratório de Malacologia, Departamento de Biologia, Universidade Federal de Santa Maria, 97.105-900 Santa Maria, RS. (modrizralok@hotmail.com)

3. Laboratório de Zoologia de Crustáceos Decápodos, Departamento de Ciências Morfobiológicas, Fundação Universidade de Rio Grande, 96.201-900 Rio Grande, RS. (paulorieger@yahoo.com.br)

\begin{abstract}
Occupation of gastropod shells by hermit crabs (Decapoda, Anomura) in the littoral of Rio Grande, Rio Grande do Sul, Brazil. The present study aimed to characterize the shell occupation by hermit crabs at the Rio Grande city, state of Rio Grande do Sul. Animals were sampled at 14 radials in Rio Grande, between 12 and 50 meters depth. Each hermit crab and its respective shell were identified, weighted and measured. A total of 408 animals were captured, of families Paguridae and Diogenidae; the most abundant species were Dardanus insignis (de Saussure, 1858) and Loxopagurus loxochelis (Moreira, 1901). The animals occupied shells from 13 gastropod species, mainly of Buccinanops lamarckii (Kiener, 1834) and B. gradatum (Deshayes, 1844). Dardanus insignis utilized shells from 12 of the 13 mollusks species registered; L. loxochelis from nine ones. In a general way, the shell occupation patterns present a correlation between the hermit crab size and the shell size, in the case of the two most abundant species the strongest correlation was between their size/weight and shell aperture width, evidencing that shells occupation is given not only by their local availability, but also by the relationship between hermit crabs variables and gastropod shells.
\end{abstract}

KEYWORDS. Shell preference, Dardanus insignis, Loxopagurus loxochelis, south Brazilian coast.

RESUMO. O presente estudo teve como objetivo caracterizar a ocupação de conchas por ermitões no litoral da cidade de Rio Grande, Estado do Rio Grande do Sul. Os animais foram amostrados em 14 radiais em Rio Grande, entre 12 e 50 metros de profundidade. Cada ermitão e sua respectiva concha foram identificados, pesados e medidos. Um total de 408 animais foi capturado, pertencentes às famílias Diogenidae e Paguridae; as duas espécies mais abundantes foram Dardanus insignis (de Saussure, 1858) e Loxopagurus loxochelis (Moreira, 1901). Os ermitões ocuparam conchas de 13 espécies de gastrópodes, principalmente de Buccinanops lamarckii (Kiener, 1834) e B. gradatum (Deshayes, 1844). Dardanus insignis utilizou 12 das 13 espécies de moluscos registradas; Loxopagurus loxochelis utilizou nove. De um modo geral, o padrão de ocupação de conchas apresenta uma correlação entre o tamanho do ermitão e o tamanho da concha; no caso das duas espécies de ermitões mais abundantes, a maior correlação foi entre peso/tamanho do animal e o tamanho da abertura da concha, evidenciando, que a ocupação de conchas se dá não apenas pela disponibilidade local das mesmas, mas também pelas relações entre as variáveis dos ermitões e das conchas de gastrópodes.

PALAVRAS-CHAVE. Preferência de conchas, Dardanus insignis, Loxopagurus loxochelis, costa sul do Brasil.

Anomura é uma infra-ordem constituída por um grupo variado de formas, algumas das quais se assemelham aos caranguejos (KAESTNER, 1970); compreende cerca de 1.400 espécies, das quais mais de 800 são representadas pelos ermitões (HAZLETT, 1981; INGLE, 1993).

Os ermitões são agrupados na superfamília Paguroidea, atualmente composta por sete famílias: Coenobitidae, Diogenidae, Lithodidae, Paguridae, Parapaguridae, Pylochelidae e Pylojacquesidae (MARTIN \& DaVIS, 2001; McLaughlin \& Lemaitre, 2001).

Uma característica peculiar desses animais é a utilização de conchas de gastrópodes como abrigo ao seu abdome não-calcificado; portanto, a concha é essencial para os ermitões, principalmente para proteger o corpo contra predadores (REESE, 1962; BERTNESS, 1982), estresse físico (REESE, 1969) e abrasões mecânicas (Bollay, 1964). A mobilidade e a proteção proporcionadas por elas devem contribuir para o fato de esses crustáceos serem encontrados em quase todo ambiente marinho (HAZLETT, 1981), apresentando uma considerável extensão batimétrica, desde a região intertidal até a sublitoral (LANCASTER, 1988).

O padrão de utilização de conchas varia entre populações de ermitões e é influenciado principalmente pelo tamanho e forma das conchas disponíveis durante a inspeção; pela localidade (área intertidal ou sublitoral) e pela preferência dos ermitões (VANCE, 1972a; Fotheringham, 1976a,b; KellogG, 1976; Scully, 1979; Bertness, 1980; Mantelatto \& Garcia, 2000; MantelatTo \& Dominciano, 2002). A escolha ou a rejeição de uma concha pelos ermitões envolve comportamentos exploratórios específicos, pelos quais estes animais analisam seu estado de conservação, tamanho, forma, peso, dimensão de sua abertura, coloração e até mesmo volume interno (Mitchell, 1976).

Além das características das conchas, outro fator importante durante a seleção é a abundância local relativa. Os fatores mencionados destas podem atuar isoladamente ou em conjunto (REESE, 1962; ConOver, 1978).

No litoral do Rio Grande do Sul são registradas seis espécies de ermitões: Dardanus insignis (de 
Saussure, 1858), Loxopagurus loxochelis (Moreira, 1901), Paguristes robustus Forest \& Saint Laurent, 1967 (Diogenidae); Pagurus exilis (Benedict, 1892), P. criniticornis (Dana, 1852) e Rhodochirus rosaceus (A. Milne Edwards \& Bouvier, 1893) (Paguridae) (RIEGER, 1999).

Apesar da incontestável relevância e destaque no contexto evolutivo, poucos trabalhos têm-se dedicado ao estudo de ermitões no litoral sul do Brasil (e. g. HeBLING \& RIEGER (1986) e RIEGER \& D'INCAO (1991)).

Neste estudo objetiva-se fornecer dados sobre alguns aspectos biológicos dos ermitões da região costeira do Rio Grande do Sul, bem como apresentar algumas características das relações morfométricas entre os ermitões e as conchas por eles ocupadas.

\section{MATERIAL E MÉTODOS}

Os ermitões e as conchas foram capturados com uma rede do tipo "beam trawl" com três metros de abertura, dez metros de comprimento, malha de $1 \mathrm{~cm}$ de entre-nós na panagem e $0,5 \mathrm{~cm}$ no saco. As amostragens foram feitas na costa de Rio Grande, Rio Grande do Sul, em 14 radiais a partir da foz da laguna dos Patos, a uma velocidade de três nós, entre 12 e $50 \mathrm{~m}$ de profundidade, no mês de dezembro de 1999 (Tab. I).

Após cada arrasto, todos os ermitões e conchas de gastrópodes foram acondicionados em caixas térmicas contendo gelo e transportados para o laboratório, onde foram congelados $\left(-20^{\circ} \mathrm{C}\right.$, em freezer) até o momento das análises. Os espécimes coletados foram depositados no Laboratório de Carcinologia da Universidade Federal de Santa Maria (UFSM), sob os lotes números 82 e 109.

Os ermitões foram descongelados à temperatura ambiente e retirados manualmente de suas conchas. Quando necessário, as conchas foram quebradas com auxílio de uma morsa. Em seguida, os exemplares foram contados, pesados a fresco em balança eletrônica (precisão $0,01 \mathrm{~g}$ ) e foi feita a caracterização do sexo considerando-se a posição dos gonóporos sob estereomicroscópio (fêmeas, na base do $3^{\circ}$ par de pereiópodos; machos, na base do $5^{\circ}$ par de pereiópodos).

Tabela I. Localização, profundidade e duração de cada arrasto em cada radial de amostragem dos ermitões coletados no litoral de Rio Grande, Rio Grande do Sul, em dezembro de 1999. (As radiais foram ordenadas apenas em função de suas profundidades).

\begin{tabular}{|c|c|c|c|}
\hline Radial & Coordenadas & $\begin{array}{l}\text { Profundidade } \\
\text { (m) }\end{array}$ & $\begin{array}{l}\text { Duração do } \\
\text { arrasto (min) }\end{array}$ \\
\hline 1 & $32^{\circ} 10^{\prime} 42^{\prime \prime} \mathrm{S} ; 51^{\circ} 01^{\prime} 50^{\prime \prime} \mathrm{W}$ & 12 & 05 \\
\hline 2 & $32^{\circ} 10^{\prime} 47^{\prime \prime} \mathrm{S} ; 52^{\circ} 02^{\prime} 12^{\prime \prime} \mathrm{W}$ & 12 & 05 \\
\hline 3 & $32^{\circ} 19^{\prime} 26^{\prime \prime} \mathrm{S} ; 52^{\circ} 00^{\prime} 59^{\prime \prime} \mathrm{W}$ & 21 & 05 \\
\hline 4 & $32^{\circ} 18^{\prime} 52^{\prime \prime} \mathrm{S} ; 51^{\circ} 59^{\prime} 43^{\prime \prime} \mathrm{W}$ & 21 & 05 \\
\hline 5 & $32^{\circ} 12^{\prime} 43^{\prime \prime} \mathrm{S} ; 51^{\circ} 54^{\prime} 15^{\prime \prime} \mathrm{W}$ & 21 & 06 \\
\hline 6 & $32^{\circ} 13^{\prime} 10^{\prime \prime} \mathrm{S} ; 51^{\circ} 54^{\prime} 47^{\prime \prime} \mathrm{W}$ & 21 & 06 \\
\hline 7 & $32^{\circ} 13^{\prime} 36^{\prime \prime} \mathrm{S} ; 51^{\circ} 43^{\prime} 57^{\prime \prime} \mathrm{W}$ & 22 & 05 \\
\hline 8 & $32^{\circ} 14^{\prime} 14^{\prime \prime} \mathrm{S} ; 51^{\circ} 44^{\prime} 35^{\prime \prime} \mathrm{W}$ & 24 & 05 \\
\hline 9 & $32^{\circ} 16^{\prime} 50^{\prime \prime} \mathrm{S} ; 51^{\circ} 49^{\prime} 36^{\prime \prime} \mathrm{W}$ & 25 & 05 \\
\hline 10 & $32^{\circ} 15^{\prime} 55^{\prime \prime} \mathrm{S} ; 51^{\circ} 48^{\prime} 10^{\prime \prime} \mathrm{W}$ & 26 & 05 \\
\hline 11 & $32^{\circ} 33^{\prime} 49^{\prime \prime} \mathrm{S} ; 51^{\circ} 38^{\prime} 44^{\prime \prime} \mathrm{W}$ & 37 & 15 \\
\hline 12 & $32^{\circ} 32^{\prime} 44^{\prime \prime} \mathrm{S} ; 51^{\circ} 38^{\prime} 56^{\prime \prime} \mathrm{W}$ & 37 & 10 \\
\hline 13 & $32^{\circ} 40^{\prime} 55^{\prime \prime} \mathrm{S} ; 51^{\circ} 30^{\prime} 47^{\prime \prime} \mathrm{W}$ & 50 & 10 \\
\hline 14 & $32^{\circ} 39^{\prime} 19^{\prime \prime} \mathrm{S} ; 51^{\circ} 30^{\prime} 41^{\prime \prime} \mathrm{W}$ & 50 & 10 \\
\hline
\end{tabular}

Os exemplares foram medidos com paquímetro de precisão $(0,01 \mathrm{~mm})$ para obtenção do comprimento do escudo cefalotorácico (CEC, medido entre a extremidade do rostro até a região mediana da sutura cervical); também foi verificado o peso úmido de cada animal em balança eletrônica (precisão 0,01g) (PE). Os indivíduos foram identificados conforme HEBLING \& RIEGER (1986) e Melo (1999).

As espécies de conchas coletadas foram identificadas segundo Rios (1994). As seguintes dimensões foram verificadas com paquímetro de precisão $(0,01 \mathrm{~mm})$ : comprimento da abertura da concha (CAC, medida da distância entre o ângulo posterior da abertura e o bordo mediano do canal sifonal da concha, em $\mathrm{mm}$ ), largura da abertura (LAC, maior distância compreendida entre a margem interior da borda exterior da abertura da concha e a parede da borda interior da abertura, em $\mathrm{mm}$ ). Também foi obtido o peso seco das conchas (PSC em gramas, através da diferença entre o peso úmido e o peso obtido após as conchas serem mantidas $24 \mathrm{~h}$ em estufa a $60^{\circ} \mathrm{C}$ ).

As duas espécies mais abundantes de ermitões foram estudadas separadamente em relação ao uso das conchas.

O teste de normalidade K-S (Kolmogorov-Smirnov) $(p<0,05)$ foi empregado para caracterizar a normalidade da distribuição dos dados (tamanho dos animais, CEC) (ZAR, 1996).

Para averiguar a razão sexual foi utilizado o teste $\chi^{2}$ $(\mathrm{p}<0,05)\left(Z_{A R}, 1996\right)$. Foi empregado o teste $t(\mathrm{p}<0,05)$ para comparar as diferenças entre as médias de tamanho (CEC) entre machos, fểmeas não-ovígeras e fêmeas ovígeras (ZAR, 1996).

Para verificar possíveis existência de diferenças entre as variáveis analisadas (espécies de gastrópodes cujas conchas foram utilizadas, o sexo e o estado reprodutivo das fêmeas dos ermitões), foi aplicado o Teste Kruskall-Wallis $(\mathrm{p}<0,05)(\mathrm{ZAR}, 1996)$.

Para avaliar as relações entre as variáveis das conchas ocupadas (variável dependente: LAC, CAC, PSC) com o tamanho dos ermitões (variável independente: CEC e PE), foi utilizada regressão linear empregando a função potência (ZAR, 1996). Foi aplicado o teste de correlação de Spearman $\left(r_{s}\right)$ para verificar a existência de correlação entre os valores emparelhados de x e y $(p<0,05)$ (ZAR, 1996).

\section{RESULTADOS}

Ao longo das radiais de coleta foram capturados 408 ermitões de cinco espécies. A espécie mais abundante foi D. insignis (195 indivíduos coletados; 47,79\%), seguida por L. loxochelis (168 indivíduos; 41,17\%), $P$. robustus (35 indivíduos; $8,58 \%$ ), oito P. exilis $(1,96 \%) \mathrm{e}$ um indivíduo de $P$. criniticornis $(0,25 \%)$. Capturou-se um indivíduo não identificado por não apresentar as estruturas para a determinação $(0,25 \%)$.

Dardanus insignis foi a espécie que apresentou o maior tamanho $(\mathrm{CEC}=26,92 \mathrm{~mm})$ (Tab. II). Os espécimes de ermitões ocuparam 13 diferentes espécies de conchas, sendo Buccinanops lamarckii (Kiener, 1834) a mais ocupada (Tab. III). 
Tabela II. Valores mínimos, máximos, média $(\bar{x})$ e desvio padrão (dp) do comprimento do escudo cefalotorácico (CEC) dos ermitões coletados no litoral de Rio Grande, Rio Grande do Sul em dezembro de 1999, separados por grupo de interesse (machos, fêmeas nãoovígeras e ovígeras) ( $\mathrm{N}$, número de indivíduos; *, indivíduo não-identificado).

\begin{tabular}{|c|c|c|c|c|c|}
\hline \multirow{3}{*}{$\mathrm{CEC}(\mathrm{mm})$} & Machos & $\begin{array}{c}\text { Fêmeas não- } \\
\text { ovígeras }\end{array}$ & Fêmeas ovígeras & $\begin{array}{c}\text { Sexo não- } \\
\text { determinado }\end{array}$ & Total \\
\hline & Mín & Mín Máx & Máx & Mín Máx & Mín Máx \\
\hline & $\overline{\mathrm{x}} \pm \mathrm{dp}(\mathrm{N})$ & $\overline{\mathrm{X}} \pm \mathrm{dp}(\mathrm{N})$ & $\overline{\mathrm{X}} \pm \mathrm{dp}(\mathrm{N})$ & $\overline{\mathrm{X}} \pm \mathrm{dp}(\mathrm{N})$ & $\overline{\mathrm{X}} \pm \mathrm{dp}(\mathrm{N})$ \\
\hline \multirow[t]{2}{*}{ D. insignis* } & $3,09 \quad 26,92$ & $3,39 \quad 12,34$ & $6,98 \quad 14,21$ & $7,09 \quad 9,94$ & $3,09 \quad 26,92$ \\
\hline & $10,64 \pm 3,39$ & $6,67 \pm 3,35(25)$ & $10,10 \pm 3,22(71)$ & $8,09 \pm 1,14(05)$ & $9,89 \pm 3,41$ \\
\hline \multirow[t]{2}{*}{ L. loxochelis } & $3,50 \quad 10,27$ & $3,35 \quad 8,19$ & $4,62 \quad 7,17$ & $4,47 \quad 6,03$ & $3,35 \quad 10,27$ \\
\hline & $6,83 \pm 1,47(91)$ & $5,55 \pm 1,45(44)$ & $5,69 \pm 1,47(29)$ & $5,10 \pm 0,82(04)$ & $6,26 \pm 1,47$ \\
\hline \multirow[t]{2}{*}{ P. robustus } & 2,68 & 8,32 & 7,74 & 5,75 & 8,32 \\
\hline & $6,86 \pm 1,85(13)$ & $6,71 \pm 1,76(20)$ & $7,74(01)$ & $5,75(01)$ & $6,77 \pm 1,30$ \\
\hline P. criniticornis & $8,73(01)$ & & & & \\
\hline \multirow{2}{*}{ P. exilis } & $4,34 \quad 6,18$ & 6,39 & & 6,91 & 6,91 \\
\hline & $5,26 \pm 2,25(02)$ & $5,66 \pm 3,12(03)$ & & $5,55 \pm 1,91(03)$ & $5,52 \pm 1,06$ \\
\hline
\end{tabular}

Tabela III. Número e porcentagem das espécies de gastrópodes cujas conchas foram utilizadas pelos ermitões no litoral de Rio Grande, Rio Grande do Sul, conforme o sexo e o estado reprodutivo das fêmeas (não-ovígeras e ovígeras) coletadas em dezembro de 1999.

\begin{tabular}{|c|c|c|c|c|c|c|c|c|c|c|}
\hline \multirow[t]{2}{*}{ Conchas ocupadas } & \multicolumn{2}{|c|}{ Machos } & \multicolumn{2}{|c|}{$\begin{array}{l}\text { Fêmeas não- } \\
\text { ovígeras }\end{array}$} & \multicolumn{2}{|c|}{ Fêmeas ovígeras } & \multicolumn{2}{|c|}{$\begin{array}{c}\text { Sexo não } \\
\text { identificado }\end{array}$} & \multicolumn{2}{|c|}{ Total } \\
\hline & $\mathrm{N}$ & $\%$ & $\mathrm{~N}$ & $\%$ & $\mathrm{~N}$ & $\%$ & $\mathrm{~N}$ & $\%$ & $\mathrm{~N}$ & $\%$ \\
\hline Buccinanops lamarckii (Kiener, 1834) & 82 & 20,10 & 27 & 6,61 & 69 & 16,90 & 8 & 1,96 & 186 & 45,57 \\
\hline Buccinanops gradatum (Deshayes, 1844) & 38 & 9,30 & 17 & 4,17 & 13 & 3,18 & 1 & 0,25 & 69 & 16,90 \\
\hline Olivancillaria urceus (Röding, 1798) & 19 & 4,65 & 17 & 4,17 & 2 & 0,49 & 2 & 0,49 & 40 & 9,80 \\
\hline Olivancillaria sp. & 16 & 3,92 & 11 & 2,69 & 1 & 0,25 & 1 & 0,25 & 29 & 7,11 \\
\hline Stramonita haemastoma (Linnaeus, 1767) & 4 & 0,98 & 9 & 2,21 & 4 & 0,98 & & & 17 & 4,17 \\
\hline Não identificadas & 14 & 3,43 & 3 & 0,74 & & & & & 17 & 4,17 \\
\hline Phalium granulatum (Born, 1778) & 6 & 1,46 & 3 & 0,74 & 5 & 1,22 & & & 14 & 3,43 \\
\hline Cymatium sp. & 6 & 1,46 & 1 & 0,25 & 3 & 0,74 & & & 10 & 2,45 \\
\hline Adelomelon brasiliana (Lamarck, 1811) & 7 & 1,71 & 1 & 0,25 & 1 & 0,25 & & & 9 & 2,21 \\
\hline Tonna galea (Linnaeus, 1758) & 6 & 1,46 & & & 1 & 0,25 & & & 7 & 1,72 \\
\hline Zidona dufresnei (Donovan, 1823) & 2 & 0,48 & & & 3 & 0,74 & & & 5 & 1,22 \\
\hline Natica sp. & 1 & 0,25 & 1 & 0,25 & & & 1 & 0,25 & 3 & 0,75 \\
\hline Conus sp. & & & 1 & 0,25 & & & & & 1 & 0,25 \\
\hline Voluta sp. & & & 1 & 0,25 & & & & & 1 & 0,25 \\
\hline Total & 201 & 49,22 & 92 & 22,58 & 101 & 24,75 & 14 & 3,45 & 408 & 100 \\
\hline
\end{tabular}

A análise dos dados, a partir do comprimento do escudo cefalotorácico, demonstrou uma distribuição nãonormal $(\mathrm{d}=0,079 ; \mathrm{p}<0,05)$ para os ermitões.

Dos 195 indivíduos coletados de Dardanus insignis, 94 eram machos $(48,21 \%), 25$ fêmeas nãoovígeras $(12,82 \%), 71$ fêmeas ovígeras $(36,41 \%)$. Para cinco indivíduos o sexo não pôde ser determinado $(2,56 \%)$. Não houve diferença no número de machos em relação às fêmeas $(0,98: 1)\left(\chi^{2}=0,02 ; p>0,05\right)$. Não foram encontradas diferenças significativas entre a média de tamanho de machos e de fêmeas (tanto não-ovígeras quanto ovígeras). Porém, as fêmeas ovígeras foram significativamente maiores que as não-ovígeras $(t=-2,77$, $\mathrm{p}<0,05)$.

Dardanus insignis ocupou conchas de 12 das 13 espécies de gastrópodes (Fig.1) encontradas em Rio Grande (RS) (Tab. III). A exceção foi Conus sp., ocupada apenas por $P$. robustus. As conchas que apresentaram o maior percentual de ocupação pertencem ao gastrópode B. lamarckii $(56,41 \%)$.

Os machos utilizaram uma maior diversidade de espécies (10), seguidos pelas fêmeas não-ovígeras (nove) e fêmeas ovígeras (oito) (mas todos ocupando predominantemente $B$. lamarckii; Fig. 1). Observou-se diferença significativa entre os tamanhos das conchas utilizadas por machos e fêmeas não-ovígeras, e também por fêmeas não-ovígeras e fêmeas ovígeras $(\mathrm{H}=21.836$, $\mathrm{p}<0,001)$. Os machos ocuparam conchas com tamanho (CAC e LAC) e peso (PSC) significativamente maior do que as utilizadas por fêmeas não-ovígeras $(\mathrm{p}<0,05)$, mas similar às habitadas por fêmeas ovígeras; fêmeas nãoovígeras ocuparam conchas menores e mais leves do que aquelas ocupadas pelas não-ovígeras (p<0,05) (Tab. IV).

Todas as regressões entre tamanho e peso de $D$. insignis e as variáveis das conchas (CAC, LAC e PSC) foram significativas $(\mathrm{p}<0,05)$ quando as conchas de todas as espécies de gastrópodes foram analisadas conjuntamente. Os melhores coeficientes de correlação $\left(r_{s}\right)$ obtidos foram entre LAC x PE $\left(\mathrm{LAC}=0,005 . \mathrm{PE}^{2,51}\right.$; $\left.r_{s}=0,798\right)$ e LAC x CEC (LAC=1,24. $\left.\mathrm{CEC}^{0,76} ; r_{\mathrm{s}}=0,832\right)$, ou seja, a largura da abertura da concha demonstrando o maior ajuste ao tamanho e peso do ermitão.

Também foram correlacionadas as medidas do animal com as da espécie de gastrópode cuja concha foi 
mais ocupada (B. lamarckii), sendo que a variável LAC também apresentou o maior ajuste ao tamanho do ermitão $\left(\mathrm{LAC}=1,56 . \mathrm{CEC}^{0,68} ; \mathrm{r}_{\mathrm{s}}=0,719\right)$.

Dos 168 indivíduos coletados de Loxopagurus loxochelis, 91 eram machos (54,17\%), 44 fêmeas nãoovígeras (26,19\%), 29 fêmeas ovígeras $(17,26 \%)$ e para quatro indivíduos o sexo não pôde ser determinado $(2,38 \%)$. A razão sexual obtida foi de 1,25 machos para cada fêmea amostrada, não diferindo estatisticamente do $1: 1\left(\chi^{2}=1,97 ; p>0,05\right)$. Os machos foram significativamente maiores (CEC) do que as fêmeas (tanto não-ovígeras como ovígeras) ( $t=3,88$ e $t=3,40$, respectivamente; $\mathrm{p}<0,05)$; fêmeas (não-ovígeras e ovígeras) não apresentaram diferenças estatísticas com relação ao tamanho.

Loxopagurus loxochelis utilizou menor número de espécies de conchas (nove) quando comparado a Dardanus insignis, porém esta diferença não foi significativa $\left(\chi^{2}=0,45 ; p>0,05\right)$. Além das espécies de gastrópodes citadas (Fig. 2), um indivíduo com sexo nãodeterminado utilizou uma concha de Natica sp. A maior

Tabela IV. Comparação entre as médias das dimensões das conchas de gastrópodes ocupadas por Dardanus insignis (de Saussure, 1858) para cada grupo de interesse (machos, fêmeas não-ovígeras e ovígeras) no litoral de Rio Grande, Rio Grande do Sul ( $\bar{x}$, média e dp, desvio padrão; CAC, comprimento da abertura da concha; LAC, largura da abertura da concha; PSC, peso seco da concha) em dezembro de 1999 (valores seguidos de letras diferentes apresentaram diferença estatística; Kruskall-Wallis, $\mathrm{p}<0,05)$.

\begin{tabular}{lccc}
\hline $\begin{array}{l}\text { Dimensões } \\
\text { da concha }\end{array}$ & Machos & $\begin{array}{c}\text { Fêmeas } \\
\text { não-ovígeras } \\
\overline{\mathrm{x}} \pm \mathrm{dp}\end{array}$ & $\begin{array}{c}\text { Fêmeas ovígeras } \\
\overline{\mathrm{x}} \pm \mathrm{dp}\end{array}$ \\
\hline CAC (mm) & $39,48 \pm 15,66^{\mathrm{a}}$ & $24,64 \pm 15,79^{\mathrm{b}}$ & $34,79 \pm 15,45^{\mathrm{a}, \mathrm{c}}$ \\
LAC (mm) & $16,12 \pm 5,89^{\mathrm{a}}$ & $10,24 \pm 5,69^{\mathrm{b}}$ & $14,37 \pm 5,5^{\mathrm{a}, \mathrm{c}}$ \\
PSC (g) & $18,59 \pm 17,66^{\mathrm{a}}$ & $6,30 \pm 18,34^{\mathrm{b}}$ & $11,57 \pm 18,11^{\mathrm{a}, \mathrm{c}}$ \\
\hline
\end{tabular}

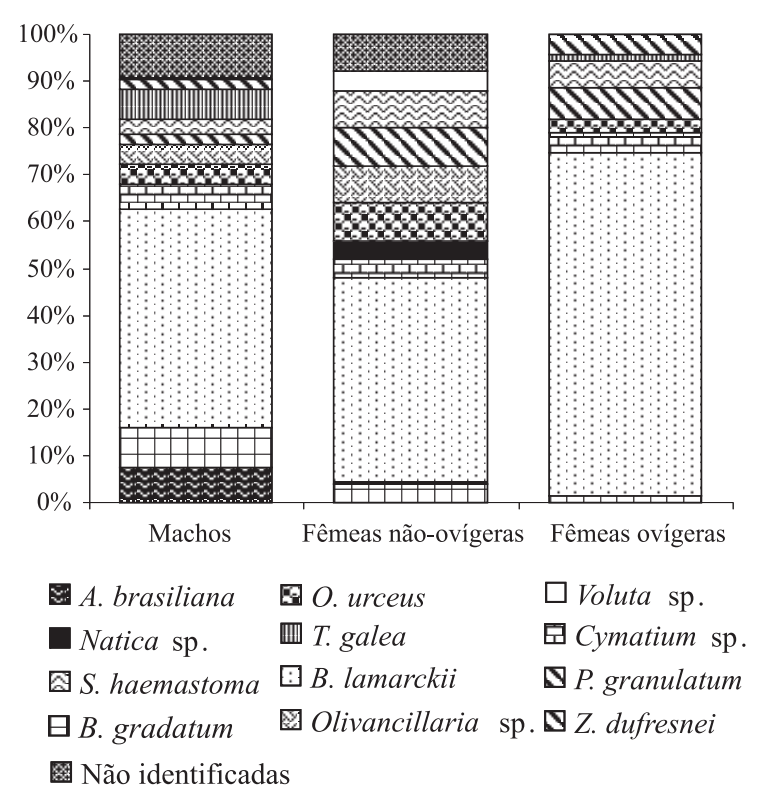

Fig. 1. Ocupação de conchas de gastrópodes por machos, fêmeas não-ovígeras e ovígeras de Dardanus insignis (de Saussure, 1858) no litoral de Rio Grande, Rio Grande do Sul, em dezembro de 1999. taxa de ocupação foi observada para as conchas de Buccinanops, sendo a mais ocupada B. lamarckii $(40,48 \%)$, seguida por B. gradatum $(33,93 \%)$.

Quando avaliada a ocupação em função do sexo, verificou-se que tanto machos como fêmeas não-ovígeras ocuparam sete diferentes espécies de gastrópodes, enquanto as fêmeas ovígeras utilizaram apenas as duas espécies com o maior percentual de ocupação $(B$. gradatum e B. lamarckii) (Fig. 2).

Houve diferença significativa entre todas as variáveis das conchas ocupadas (CAC, LAC e PSC) entre os sexos $(\mathrm{H}=40.660, \mathrm{p}<0,001)$; exceção foi em relação às fêmeas não-ovígeras e ovígeras, que não apresentaram diferença significativa $(\mathrm{p}>0,05)(\mathrm{Tab} . \mathrm{V})$.

Quando relacionado às variáveis de todas as conchas ocupadas, L. loxochelis apresentou o maior ajuste à $\mathrm{LAC}\left(\mathrm{LAC}=8,32 . \mathrm{PE}^{0,27} ; \mathrm{r}=0,854\right)$. O mesmo foi verificado para as duas espécies de conchas mais ocupadas, $B$. lamarckii $\left(\mathrm{LAC}=8,14 \cdot \mathrm{PE}^{0,34} ; \mathrm{r}_{\mathrm{s}}=0,896\right)$ e $B$. gradatum $\left(\mathrm{LAC}=8,38 . \mathrm{PE}^{0,24} ; \mathrm{r}_{\mathrm{s}}=0,818\right)$.

Tabela V. Comparação entre as médias das dimensões das conchas de gastrópodes ocupadas por Loxopagurus loxochelis (Moreira, 1901) para cada grupo de interesse (machos, fêmeas não-ovígeras e ovígeras) no litoral de Rio Grande, Rio Grande do Sul ( $\bar{x}$, média e dp, desvio padrão; CAC, comprimento da abertura da concha; LAC, largura da abertura da concha; PSC, peso seco da concha) em dezembro de 1999 (valores seguidos de letras diferentes apresentaram diferença estatística; Kruskall-Wallis, $\mathrm{p}<0,05$ ).

\begin{tabular}{lccc}
\hline $\begin{array}{l}\text { Dimensões } \\
\text { da concha }\end{array}$ & Machos & $\begin{array}{c}\text { Fêmeas } \\
\text { não-ovígeras } \\
\overline{\mathrm{x}} \pm \mathrm{dp}\end{array}$ & $\begin{array}{c}\text { Fêmeas ovígeras } \\
\overline{\mathrm{x}} \pm \mathrm{dp}\end{array}$ \\
\hline CAC $(\mathrm{mm})$ & $25,73 \pm 6,62^{\mathrm{a}}$ & $20,75 \pm 6,62^{\mathrm{b}}$ & $17,63 \pm 6,77^{\mathrm{b}, \mathrm{c}}$ \\
LAC (mm) & $11,00 \pm 2,86^{\mathrm{a}}$ & $8,39 \pm 2,89^{\mathrm{b}}$ & $8,04 \pm 2,76^{\mathrm{b}, \mathrm{c}}$ \\
PSC $(\mathrm{g})$ & $6,56 \pm 3,40^{\mathrm{a}}$ & $3,92 \pm 3,44^{\mathrm{b}}$ & $2,46 \pm 3,62^{\mathrm{b}, \mathrm{c}}$ \\
\hline
\end{tabular}

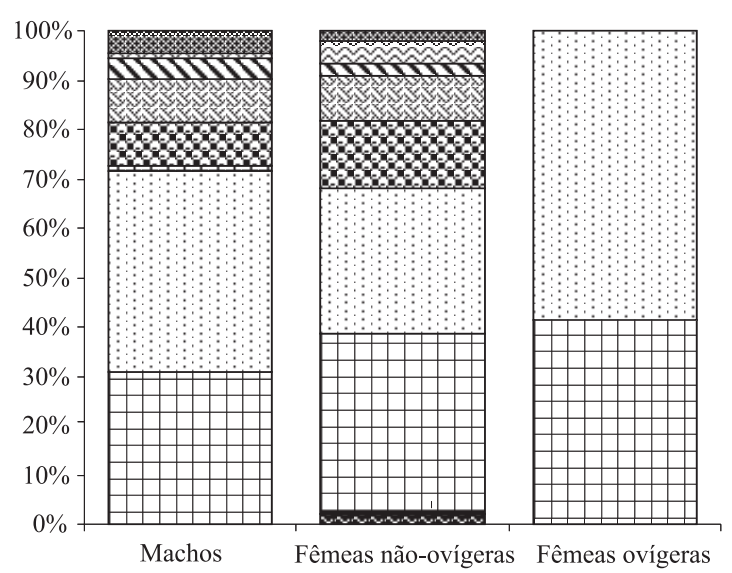

\begin{tabular}{|c|c|}
\hline A. brasiliana & 요 O.urceus \\
\hline 国 Cymatium sp. & 图 S. haemastona \\
\hline $\mathbf{\nabla}$. granulatum & $\square$ B. lamarckii \\
\hline 日B. gradatum & Olivancillaria sp. \\
\hline
\end{tabular}

Fig. 2. Ocupação de conchas de gastrópodes por machos, fêmeas não-ovígeras e ovígeras de Loxopagurus loxochelis (Moreira, 1901) no litoral de Rio Grande, Rio Grande do Sul em dezembro de 1999. 


\section{DISCUSSÃO}

Foram encontradas cinco das seis espécies de ermitões registradas para o Estado do Rio Grande do Sul. A única espécie não amostrada foi $R$. rosaceus, que ocorre em profundidades entre 100 - 209m (RIEGER 1997).

As espécies mais abundantes foram $D$. insignis e L. loxochelis, ambas típicas do Oceano Atlântico ocidental que ocorrem a profundidades que variam de 1,5 a $500 \mathrm{~m}$ e de 8 a 30m, respectivamente (RIEGER, 1997).

O dimorfismo sexual em relação ao tamanho é um padrão em decápodos, sendo machos geralmente maiores (ABRAMs, 1988). Esse padrão também foi registrado para diversas espécies de ermitões, principalmente no litoral paulista, onde existe um maior número de estudos (Negreiros-Fransozo et al., 1991; Negreiros-Fransozo \& FransOzo, 1992; ReIGADA \& SANTOS, 1997; FransOzo \& Mantelatto, 1998; Turra \& Leite, 1999; Bertini \& Fransozo, 2000; Mantelatto \& Sousa, 2000; Garcia \& Mantelatto, 2001; Martinelli et al., 2002 e Bertini et al., 2004).

Nos espécimes de $D$. insignis deste estudo não foi verificada diferença no tamanho (CEC) entre machos e fêmeas (não-ovígeras e ovígeras), diferentemente do observado por BRANCO et al. (2002) ao estudar a espécie em Santa Catarina (machos maiores).

Para L. loxochelis, os machos atingiram maior tamanho que as fêmeas, como também verificado no litoral norte do Estado de São Paulo por MARTinelli et al. (2002) e BERTINI et al. (2004).

Segundo Abrams (1988), três fatores parecem ser responsáveis pelo dimorfismo sexual de L. loxochelis e outros crustáceos: o direcionamento diferencial de energia para o crescimento; a seleção sexual (onde machos maiores apresentam maior sucesso na obtenção de fêmeas para a cópula); e prioridade na escolha da concha mais adequada, com machos maiores alcançando maior sucesso na obtenção de conchas com características mais apropriadas, podendo assim competir com maior eficácia por outros recursos.

Embora para D. insignis não se tenha encontrado diferença significativa no tamanho, não se descarta a hipótese de que esta exista, visto que no presente estudo apenas uma coleta foi realizada. Amostragens periódicas viabilizariam afirmações mais fidedignas quanto aos padrões populacionais das diferentes espécies. Com efeito, os machos atingem tamanhos maiores que as fêmeas [e. g. Clibanarius vittatus (Bosc, 1802) (REIGADA \& Santos, 1997), Paguristes erythrops Holthuis, 1959 (Garcia \& Mantelatto, 2001), Calcinus tibicen (Herbst, 1791) (Fransozo et al., 2003), Pagurus brevidactylus (Stimpson, 1859) (MANTELATTO et al., 2005)] o que parece ser um padrão usual entre os ermitões da costa brasileira.

$\mathrm{Na}$ natureza muitas populações e guildas são provavelmente limitadas por um único recurso, mas, muitas destas por uma grande quantidade de fatores. Outrossim, em algumas guildas, um recurso pode ser muito mais importante do que todos os outros fatores em restringir o crescimento populacional (VANCE, 1972b). Para populações de ermitões, a concha parece exercer esse efeito.

Loxopagurus loxochelis foi encontrado em nove espécies de conchas no presente estudo. Porém, em
Ubatuba, litoral de São Paulo, foi encontrado em seis espécies (Martinelli \& Mantelatto, 1999). Dessas espécies, apenas três também foram utilizadas no litoral do Rio Grande do Sul: B. gradatum, O. urceus e $S$. haemastoma. Já $D$. insignis foi registrado em 12 espécies de conchas de gastrópodes nesse trabalho.

Em revisão realizada por RIEGER (1997), o autor assinalou $D$. insignis ocupando freqüentemente quatro espécies de conchas (Adelomelon brasiliana, Tonna galea, Buccinanops gradatum e Olivancillaria urceus), todas também ocupadas pela espécie no presente estudo.

De acordo com Scully (1979), indivíduos de duas populações têm diferenças significativas na preferência por conchas como resultado de peculiaridades nos fatores físicos do ambiente, seu efeito direto sobre custo metabólico de ermitões habitando aquele ambiente, e ainda, efeito indireto na qualidade e abundância de conchas de gastrópodes. Nesse sentido, a disponibilidade local de conchas de gastrópodes parece exercer um efeito crucial sobre a ocupação dessas por diferentes populações de ermitões.

As análises do presente estudo demonstraram que para $D$. insignis os machos ocuparam uma maior diversidade de conchas que as fêmeas (e também conchas maiores e mais pesadas do que as fêmeas não-ovígeras). Para L. loxochelis, fêmeas ovígeras ocuparam apenas duas espécies de conchas, enquanto machos e fêmeas nãoovígeras utilizaram sete espécies; os machos também utilizaram conchas maiores e mais pesadas do que as fêmeas (não-ovígeras e ovígeras). Possivelmente machos despendem maior tempo e esforço na busca por conchas. Adicionalmente, devido ao maior tamanho alcançado por estes, os mesmos podem ser favorecidos em possíveis combates intra-específicos por conchas.

Conchas mais leves e com maior volume interno favorecem às fêmeas ovígeras, visto que o uso desse tipo de concha proporciona maior espaço interno para os ovos (FotheringHAM, 1976b), além de maior facilidade de deslocamento (em função de um peso menor), enquanto as mais pesadas favorecem aos machos; segundo GHERARDI (1991), os machos estariam mais expostos à predação durante a competição por fêmeas, e as conchas mais pesadas serviriam como proteção adicional a esses animais. Para fêmeas ovígeras, o uso de conchas "sub-ótimas" pode reduzir o sucesso reprodutivo (tanto conchas maiores que o tamanho considerado "ótimo" como conchas menores podem ter esse efeito). Considerando conchas maiores, as fêmeas ovígeras teriam de despender mais energia na manutenção da concha em competição, dificultando a busca por alimento (FotheringhAM, 1976a) (pois conchas grandes estão menos disponíveis no ambiente e os machos maiores são competidores adicionais). Estas poderiam ser a razão pela qual as fêmeas ovígeras das duas espécies ocupam menor diversidade de conchas do que machos e fêmeas não-ovígeras.

Fato importante a ser destacado é que a disponibilidade de conchas na natureza é determinada pela abundância relativa de gastrópodes vivos e suas taxas de mortalidade (Meireles et al., 2003). Portanto, estudos sobre a disponibilidade de conchas no ambiente são extremamente importantes para explanar sobre o 
padrão de ocupação por ermitões. Contudo, existe uma carência de publicações que abordem a disponibilidade de conchas de gastrópodes.

Foi observado que a largura da abertura da concha foi a que melhor refletiu a associação com os ermitões. Nos estudos realizados no litoral de São Paulo, Calcinus tibicen apresentou melhores correlações entre o tamanho e o peso dos ermitões com o peso da concha (MANTELATTO \& GARCIA, 2000), Paguristes tortugae Schmitt, 1933 entre o peso do animal e o peso e o volume interno das conchas (Dominciano \& Mantelatto, 2004); no estudo com $L$. loxochelis, as melhores relações foram entre o comprimento do própodo quelar esquerdo e o comprimento da abertura da concha (MARTINELli \& Mantelatto, 1999).

Segundo Conover (1978), Pagurus pollicaris Say, 1817 seleciona as conchas em função do peso e do volume, dependendo da espécie de concha. Em experimentos de laboratório, o volume foi a variável que apresentou maior importância. Assim, corrobora-se a hipótese de que o uso de conchas se dê não só pela disponibilidade destas no ambiente, mas também pela adequação dos ermitões as mesmas.

Ermitões em conchas menores do que os tamanhos preferidos crescem menos e têm proles menores do que ermitões em tipos de conchas idênticas, mas de tamanho ideal. Por conseguinte, a disponibilidade de conchas pode afetar o crescimento e a reprodução do ermitão (BERTNESS, 1981).

Esse estudo demonstrou que a utilização de conchas pelas duas espécies de ermitões mais abundantes na área amostrada segue o padrão encontrado pelas mesmas espécies em estudos realizados em outros locais do país, mesmo realizando-se apenas dois dias amostragem. Com exceção de notas de ocorrências e distribuição de espécies, poucos estudos foram realizados no litoral sul do Brasil, sendo que a continuação deste trabalho é de fundamental importância para contribuir com o conhecimento da biologia destas espécies.

\section{REFERÊNCIAS BIBLIOGRÁFICAS}

Abrams, P. A. 1988. Sexual difference in resource use in hermit crabs; consequences and causes. In: ChelazzI, G. \& VANNini, M. eds. Behavioral adaptation to intertidal life. New York, Plenum. 283-296p.

Bertini, G. \& Fransozo, A. 2000. Population dynamics of Petrochirus diogenes (Crustacea, Anomura, Diogenidae) in Ubatuba Region, São Paulo, Brazil. Crustacean Issues 12:331-342.

Bertini, G.; Fransozo, A. \& Braga, A. A. 2004. Ecological distribution and reproductive period of the hermit crab Loxopagurus loxochelis (Anomura, Diogenidae) on the northern coast of São Paulo State, Brazil. Journal of Natural History 38(18):2331-2344.

Bertness, M. D. 1980. Shell preference and utilization patterns in littoral hermit crabs of the bay of Panama. Journal of Experimental Marine Biology and Ecology 40:1-16. 1981. The influence of shell-type on hermit crab growth rate and clutch size. Crustaceana 40:197-205.

1982. Shell utilization, predation pressure, and thermal stress in Panamanian hermit crabs: an interoceanic comparison. Journal of Experimental Marine Biology and Ecology 64:159-187.

Bollay, M. 1964. Distribution and utilization of gastropod shells by the hermit crabs Pagurus samuelis, Pagurus granosimanus, and Pagurus hirsutiusculus at Pacific Grove, California. Veliger 6:71-76.
Branco, J. O.; Turra, A. \& Souto, F. X. 2002. Population biology and growth of the hermit crab Dardanus insignis at Armação do Itapocoroy, southern Brazil. Journal of the Marine Biological Association of the United Kingdom 82:597-603.

Conover, M. R. 1978. The importance of various shell characteristics to the shell - selection behavior on the hermit crabs. Journal of Experimental Marine Biology and Ecology 32:131-142.

Dominciano, L. C. C. \& Mantelatto, F. L. M. 2004. The influence of shell species and size on the shell selection pattern of Paguristes tortugae (Decapoda, Diogenidae) from Anchieta Island (Ubatuba, Brazil). Iheringia, Série Zoologia, 94(4):425-428.

Fotheringham, N. 1976a. Hermit crabs as a limiting resource (Decapoda, Paguridae). Crustaceana 31(2):193-199.

. 1976b. Population consequences of shell utilization by hermit crabs. Ecology 57(3):570-578.

Fransozo, A. \& Mantelatto, F. L. M. 1998. Population structure and reproductive period of the hermit crab Calcinus tibicen (Decapoda: Diogenidae) in the region of Ubatuba, São Paulo, Brazil. Journal of Crustacean Biology 18(4):738-745.

Fransozo, A.; Garcia, R. B. \& Mantelatto, F. L. M. 2003. Morphometry and sexual maturity of the tropical hermit crab Calcinus tibicen (Crustacea, Anomura) from Brazil. Journal of Natural History 37(3):297-304.

Garcia, R. B. \& Mantelatto, F. L. M. 2001. Population dynamics of the hermit crab Paguristes erythrops (Diogenidae) from Anchieta Island, southern Brazil. Journal of the Marine Biological Association of the United Kingdom 81:955-960.

Gherardi, F. 1991. Relative growth, population structure, and shell-utilization of the hermit crab Clibanarius erythropus in the Mediterranean. Oebalia 17:181-196.

Hazlett, B. A. 1981. The behavioral ecology of hermit crab. Annual Review of Ecology and Systematics 12:1-22.

Hebling, N. J. \& Rieger, P. J. 1986. Os ermitões (Crustacea Decapoda: Paguridae e Diogenidae) do litoral do Rio Grande do Sul, Brasil. Atlântica 8:63-77.

Ingle, R. 1993. Hermit crabs of the northeastern Atlantic Ocean and the Mediterranean Sea. London, Chapman \& Hall. 495p.

Kaestner, A. 1970. Invertebrate zoology. III. Crustacea. London, Interscience. 523p.

KellogG, C. W. 1976. Gastropod shells: a potentially limiting resource for hermit crabs. Journal of Experimental Marine Biology and Ecology 22:101-111.

LANCASter, I. 1988. Pagurus bernhardus (L.) - an introduction to the natural history of hermit crabs. Field Studies 7:189-238.

Mantelatto, F. L. M. \& Dominciano, L. C. C. 2002. Pattern of shell utilization by the hermit crab Paguristes tortugae (Diogenidae) from Anchieta Island, southern Brazil. Scientia Marina 66(3):265-272.

Mantelatto, F. L. M. \& Garcia, R. B. 2000. Shell utilization pattern of the hermit crab Calcinus tibicen (Diogenidae) from Southern Brazil. Journal of Crustacean Biology 20(3):460-467.

Mantelatto, F. L. M. \& Sousa, L. M. 2000. Population biology of the hermit crab Paguristes tortugae Schmitt, 1933 (Anomura, Diogenidae) from Anchieta Island, Ubatuba, Brazil. Nauplius 8(2): 185-193.

Mantelatto, F. L.; Christofoletti, R. A. \& Valenti, W. C. 2005. Population structure and growth of the hermit crab Pagurus brevidactylus (Anomura: Paguridae) from the northern of São Paulo, Brazil. Journal of the Marine Biological Association of the United Kingdom 85(1):127-128.

Martin, J. W. \& Davis, G. E. 2001. An updated classification of the recent Crustacea. Los Angeles, Natural History Museum of Los Angeles Country. Science Series, 39. 124p.

Martinelli, J. M. \& Mantelatto, F. L. M. 1999. Shell utilization by the hermit crab Loxopagurus loxochelis (Diogenidae) in Ubatuba Bay, Brazil. In: Schram, F. R. \& Vanpel Klein, J. C. orgs. Proceedings of the $4^{\text {th }}$ International Crustacean Congress - Crustaceans and the Biodiversity Crisis. 1ed. Leiden, Brill. v.1, p.719-731.

Martinelli, J. M.; Mantelatto, F. L. M. \& Fransozo, A. 2002. Population structure and breeding season of the South Atlantic 
hermit crab, Loxopagurus loxochelis (Anomura, Diogenidae) from the Ubatuba region, Brazil. Crustaceana 75(6):791-802.

McLaughlin, P. A. \& Lemaitre, R. 2001. A new family for a genus and new species of hermit crabs of the superfamily Paguroidea (Decapoda: Anomura) and its phylogenetic implications. Journal of Crustacean Biology 21(4):1062-1076.

Meireles, A. L.; Biagi, R. \& Mantelatto, F. L. M. 2003. Gastropod shell availability as a potential resource for the hermit crab infralittoral fauna of Anchieta Island (SP), Brazil. Nauplius 11(2):99-105.

Melo, G. A. S. 1999. Manual de identificação dos Crustacea Decapoda do litoral brasileiro: Anomura, Thalassinidea, Palinuridea e Astacidea. São Paulo, Plêiade. 551p.

Mitchell, K. A. 1976. Shell selection in the hermit crab Pagurus bernhardus. Marine Biology 35:335-346.

Negreiros-Fransozo, M. L. \& Fransozo, A. 1992. Estrutura populacional e relação com a concha em Paguristes tortugae Schmitt, 1933 (Decapoda, Diogenidae), no litoral norte do Estado de São Paulo, Brasil. Naturalia 17:31-42.

Negreiros-Fransozo, M. L.; Fransozo, A. \& Hebling, N. J. 1991. Estrutura populacional e determinação do tamanho da concha em 4 espécies de ermitões (Crustacea, Decapoda, Anomura) do litoral paulista. Biotemas 4(2):135-148.

Reese, E. 1962. Shell selection behavior of hermit crabs. Animal Behaviour 10:347-360.

1969. Behavioral adaptation of intertidal hermit crabs. American Zoologist 9:343-355.

Reigada, A. L. D. \& Santos, S. 1997. Biologia e relação com a concha em Clibanarius vittatus (Bosc, 1802) (Crustacea,
Diogenidae) em São Vicente, SP, Brasil. Brazilian Archives of Biology and Technology 40(4):941-952.

Rieger, P. J. 1997. Os "Ermitões" (Crustacea, Decapoda, Parapaguridae, Diogenidae e Paguridae) do litoral do Brasil. Nauplius 5(2):99-124.

. 1999. Famílias Diogenidae e Paguridae (ermitões). In: Buckup, L. \& Bond-Buckup, G. Os crustáceos do Rio Grande do Sul. Porto Alegre, UFRGS. p.343-361.

Rieger, P. J. \& D'Incao, F. 1991. Distribuição de larvas de Loxopagurus loxochelis (Decapoda, Diogenidae) na região adjacente à Barra de Rio Grande, RS. Nerítica 6(1-2):93-106.

Rios, E. C. 1994. Seashells of Brazil. 2ed. Rio Grande do Sul. Fundação Universidade de Rio Grande, Instituto Acqua, Museu Oceanográfico de Rio Grande, Universidade de Rio Grande. 368p.

Scully, E. P. 1979. The effects of gastropod shell availability and habitat characteristics on shell utilization by the intertidal hermit crab Pagurus longicarpus Say. Journal of Experimental Marine Biology and Ecology 37:139-152.

Turra, A. \& Leite, F. P. P. 1999. Population structure and fecundity of the hermit crab Clibanarius antillensis Stimpson, 1862 (Anomura, Diogenidae) in southeastern Brazil. Bulletin of Marine Science 64(2):281-289.

VANCE, R. R. 1972a. The role of shell adequacy in behavior interactions in hermit crabs. Ecology 53:1075-1083.

1972b. Competition and mechanisms of coexistence in three sympatric species of intertidal hermit crabs. Ecology 53:1062-1074.

ZAR, J. H. 1996. Biostatistical analysis. New Jersey, PrenticeHall. $907 p$ 